\title{
Singularities in the optical response of cuprates.
}

\author{
Ar. Abanov ${ }^{1}$, Andrey V. Chubukov ${ }^{1}$, and Jörg Schmalian ${ }^{2}$ \\ 1 Department of Physics, University of Wisconsin, Madison, WI 53706 \\ ${ }^{2}$ Department of Physics and Ames Laboratory, Iowa State University, Ames, IA 50011
}

(November 1, 2018)

\begin{abstract}
We argue that the detailed analysis of the optical response in cuprate superconductors allows one to verify the magnetic scenario of superconductivity in cuprates, as for strong coupling charge carriers to antiferromagnetic spin fluctuations, the second derivative of optical conductivity should contain detectable singularities at $2 \Delta+\Delta_{\text {spin }}, 4 \Delta$, and $2 \Delta+2 \Delta_{\text {spin }}$, where $\Delta$ is the amplitude of the superconducting gap, and $\Delta_{s}$ is the resonance energy of spin fluctuations measured in neutron scattering. We argue that there is a good chance that these singularities have already been detected in the experiments on optimally doped $Y B C O$.
\end{abstract}

PACS numbers:71.10.Ca,74.20.Fg,74.25.-q

The pairing state in cuprate superconductors is predominantly made out of Cooper pairs with $\mathrm{d}_{x^{2}-y^{2}}$ symmetry [1]. This salient universal property of all high $T_{c}$ materials entails constraints on the microscopic mechanism of superconductivity. However, it does not uniquely determine it, leading to a quest for experiments which can identify "fingerprints" of a specific microscopic mechanism of d-wave superconductivity, a strategy similar to the one used in conventional superconductors [2].

Several resent experiments were interpreted as an indirect evidence that $\mathrm{d}_{x^{2}-y^{2}}$ pairing in cuprates is produced by an exchange of collective spin fluctuations peaked at or near antiferromagnetic momentum $\mathbf{Q}=(\pi, \pi)$ [3]. In particular, the distance between the peak and the dip in the fermionic spectral function, $A_{\mathbf{k}}(\omega)$, in ARPES experiments coincides with the frequency $\Delta_{s}$ of the resonance peak measured in neutron scattering [4, 5]. This is exactly what one should expect for fermions interacting with a resonating spin collective mode [4.5] (for phonon mediated superconductors, this is known as the Holstein effect [6]). Similarly, a peak-dip structure of the SIS tunneling conductance with peak-dip distance roughly consistent with $\Delta_{s}$ has been obtained in the measurements on break junctions by Zasadzinski et al. for various doping values [7]. Carbotte et al. [8] analyzed optical conductivity $\sigma(\omega)$ in magnetically mediated $d$-wave superconductors and argued that $\Delta_{s}$ can be extracted from the measurements of the second derivative of $\sigma(\omega)$.

In this paper we reexamine the behavior of the optical conductivity in superconductors with quasiparticles strongly coupled to their own collective spin modes. Our results partly agree and partly disagree with those by Carbotte et al. [8] (see below). The key prediction of this paper, however, is novel: we argue that by measuring the frequency derivatives of the conductivity, one can not only verify the magnetic scenario, but, in principle, also independently determine both $\Delta_{s}$ and $\Delta$ in the same experiment.

Our argument goes as follows. For a superconductor, the real part of the conductivity, $\sigma_{1}(\omega)$, has a $\delta$ - func- tional piece due to the presence of the superconducting condensate. A nonzero $\sigma_{1}(\omega)$ at a finite frequency is only possible if fermions have a finite lifetime. More precisely, one of the two fermions exited in a process causing the AC conductivity should have a finite scattering rate, while another should be able to propagate, i.e., its energy should be larger than $\Delta$. For clean, phonon-mediated superconductors, there are two sources for fermionic decay (see Fig. 1). One is a direct four-fermion interaction, which yields a threshold in the imaginary part of the self-energy, $\Sigma^{\prime \prime}(\omega)$, at $\omega=3 \Delta$ - the minimal energy necessary to pull all three fermions in the final state out of the condensate of Cooper pairs. Another is the interaction between an electron and an optical phonon. It yields the onset of $\Sigma^{\prime \prime}(\omega)$ at $\omega=\Delta+\Omega_{p}$, where $\Omega_{p}$ is the frequency of an optical phonon [6] (for simplicity, we assumed that the phonon propagator has a single pole). For the values of the coupling constant used to interpret the tunneling data in strongly coupled conventional superconductors like $P b$ [9], $\Omega_{p}>2 \Delta$, i.e., the onset of conductivity is at $3 \Delta+\Delta=4 \Delta(2 \Delta$ for dirty superconductors [10]), while the signatures of phonon-assisted damping only show up at higher frequencies, and are uncorrelated with the behavior of $\sigma_{1}(\omega)$ near $4 \Delta$.
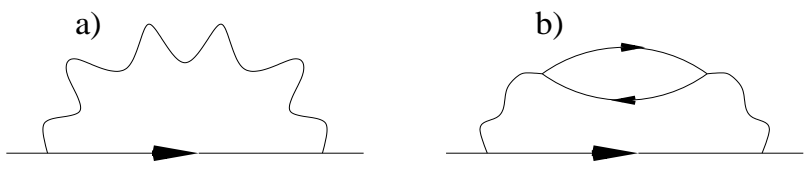

FIG. 1. a) The exchange diagram for boson mediated interaction. The solid line stands for a propagating fermion. The wiggled line is a phonon propagator in case of electronphonon interaction, and a magnon line in case of spin- fluctuation mediated interaction. b) The lowest order diagram for the fermionic self energy due to a direct four fermion interaction, also represented by a wiggly line

For spin-mediated superconductivity, the situation is different. In the one-band model for cuprates, which we adopt, the underlying interaction is solely a Hubbard- 
type four-fermion interaction. Spin excitations appear as collective modes of fermions, and their velocity $v_{s}$ is comparable to $v_{F}$. For $v_{s} \sim v_{F}$, the low frequency spin dynamics is dominated by a decay process into a particlehole pair and is purely relaxational in the normal state, with nearly featureless $\chi^{\prime \prime}(\mathbf{Q}, \omega)$ [5]. (for phonon superconductors the relaxation is also present but is strongly reduced due to a smallness of the sound velocity compared to $\left.v_{F} 11\right)$.

Below $T_{c}$, fermions acquire a gap, and spin-decay becomes impossible for energies below $2 \Delta$. The direct four-fermion interaction process in Fig. 1 $1 \mathrm{~b}$ then yields a threshold in $\Sigma^{\prime \prime}$ at $3 \Delta$ which gives rise to a singularity in the conductivity at $\omega=4 \Delta\left[12\right.$. If $\chi^{\prime \prime}(\mathbf{Q}, \omega)$ remained featureless, this would be the only effect. However, several authors have demonstrated that the residual attraction in a $d$-wave superconductor binds a particle and a hole into a spin exciton at an energy $\Delta_{s}<2 \Delta$. This effect gives rise to a peak in $\chi^{\prime \prime}(\mathbf{Q}, \omega)$ at $\omega=\Delta_{s}$ and makes it look like the spectral function for optical phonons. Accordingly, the conductivity acquires another threshold at $2 \Delta+\Delta_{s}$. Formally, this is analogous to the phonon case, but in distinction to phonons, $\Delta_{s}<2 \Delta$. Then $2 \Delta+\Delta_{s}<4 \Delta$, i.e., in clean systems, the lower threshold corresponds to the scattering by a spin exciton. Moreover, since both effects are due to the same underlying interaction, (the diagram in Fig. 1 $1 \mathrm{~b}$ is just the first term in the series of graphs which constitute the spin-mediated scattering process shown in Fig 1 1b) the ratio $\Delta_{s} / \Delta$ and the relative intensity of the singularities in $\sigma_{1}(\omega)$ at $4 \Delta$ and $2 \Delta+\Delta_{s}$ are correlated. This correlation is a "fingerprint" of the spin-fluctuation mechanism. We will argue that there are strong indications that both singularities have been observed in the measurements of the optical conductivity in YBCO [8], and their position and relative intensity are in reasonable agreement with the theory.

Before we proceed with the calculations, a comment is in order. In the above discussion we neglected the momentum dependence of the $d$-wave gap. Meanwhile, the computations of the optical conductivity involve averaging of the lifetime over the Fermi surface 13,14. It is then a'priori unclear whether the angular dependence of the $d$-wave gap with $\Delta(\theta) \propto \cos 2 \theta$ affects the positions of the two thresholds in the conductivity. Carbotte et al argued [8] that it does, and the singularity at $2 \Delta+\Delta_{s}$ (which they only considered) is determined by some averaged $|2 \Delta(\theta)| \approx \Delta$. We argue that averaging reduces strengths of the singularities but doesn't shift their positions. Our argument is two-fold. First, we explicitly demonstrate below that the singularity in the conductivity occurs at a frequency equal to the maximum value of the gap. Second, two of us and Finkel'stein argued earlier [15] that for spin-mediated $d$-wave superconductivity, $\Delta(\theta)$ is at its maximum at hot spots (points at the Fermi surface separated by $\mathbf{Q}$ ) [15]. These are precisely the Fermi points which determine the position of the excitonic pole in $\chi^{\prime \prime}(\mathbf{Q}, \omega)$. Accordingly, the singularity in conductivity entirely comes from fermions near hot spots, and the threshold frequency $2 \Delta+\Delta_{s}$ involves a maximum value of the gap and the resonance spin frequency at momentum $\mathbf{Q}$. The same argumentation implies that $4 \Delta$ threshold also involves a maximum value of the gap. Note for clarification that we are only considering here the singularities in the conductivity at $\omega>2 \Delta$. The regular part of $\sigma_{1}(\omega)$ is not necessary confined to hot spots. In particular, for $\omega \ll \Delta$ the optical response is dominated by nodal quasiparticles for which $\Sigma^{\prime \prime}$ is nonzero down to the lowest frequencies.

We now proceed with the calculations. The real part of the optical conductivity in a superconductor is given by

$$
\sigma_{1}(\omega)=\operatorname{Re} \frac{i}{\omega+i \delta} \int d \theta \Pi_{\sigma}(\theta, \omega)
$$

where $\Pi_{\sigma}(\theta, \omega)$ is the fully renormalized current-current correlator. In Matsubara frequencies, it is given by

$$
\begin{aligned}
\Pi_{\sigma}\left(i \omega_{n}\right) \propto & \frac{1}{\beta} \sum_{m} \int \frac{d^{2} k}{(2 \pi)^{2}}\left[G_{\mathbf{k}}\left(i \omega_{n}+i \omega_{m}\right) G_{\mathbf{k}}\left(i \omega_{m}\right)\right. \\
& \left.+F_{\mathbf{k}}\left(i \omega_{n}+i \omega_{m}\right) F_{\mathbf{k}}\left(i \omega_{m}\right)\right]
\end{aligned}
$$

and the normal and anomalous Green's functions are

$$
\begin{aligned}
G_{\mathbf{k}}\left(i \omega_{m}\right) & =\frac{\Sigma_{\mathbf{k}}\left(i \omega_{m}\right)+\varepsilon_{\mathbf{k}}}{\Sigma_{\mathbf{k}}^{2}\left(i \omega_{m}\right)-\Phi_{\mathbf{k}}^{2}\left(i \omega_{m}\right)-\varepsilon_{\mathbf{k}}^{2}}, \\
F_{\mathbf{k}}\left(i \omega_{m}\right) & =\frac{\Phi_{\mathbf{k}}\left(\omega_{m}\right)}{\Sigma_{\mathbf{k}}^{2}\left(i \omega_{m}\right)-\Phi_{\mathbf{k}}^{2}\left(i \omega_{m}\right)-\varepsilon_{\mathbf{k}}^{2}},
\end{aligned}
$$

(we adsorbed a bare $i \omega_{m}$ term into $\Sigma_{\mathbf{k}}\left(i \omega_{m}\right)$. In principle, $\Pi_{\sigma}$ is modified by vertex corrections related to $d \Sigma / d k$ [11], but for spin-mediated scattering these corrections are small (see below).

As an input for the computation of $\Pi_{\sigma}$ we need the forms of the fermionic self-energy $\Sigma_{\mathbf{k}}\left(i \omega_{m}\right)$ and the anomalous vertex $\Phi_{\mathbf{k}}\left(i \omega_{m}\right)$. We obtained these forms in Ref. 16 by deriving and solving a set of Eliashberg equations within the spin-fermion model. This model adequately describes the interaction between low-energy fermions and their collective spin degrees of freedom [5,15,16 at energies smaller than $E_{F}$. The full dynamical spin susceptibility peaked at (or near) $\mathbf{Q}$ mediates $d_{x^{2}-y^{2}}$ superconductivity. As discussed, this susceptibility is by itself affected by low-energy fermions via a decay process into a particle and a hole, and has to be computed together with the fermionic self-energy and the pairing vertex.

The justification of the Eliashberg approach for the spin-mediated superconductivity was discussed earlier [5, 15, 16], and we just quote the result: at strong dimensionless spin-fermion coupling $\lambda$, vertex corrections 
and $v_{F}^{-1} d \Sigma / d k_{\perp}$, where $k_{\perp}$ is the component of the momentum transverse to the Fermi surface, are small compared to $d \Sigma / d \omega$ by $\log \lambda / \lambda$. In what follows we will neglect these corrections, i.e., approximate $\Sigma_{\mathbf{k}}\left(i \omega_{m}\right)$ and $\Phi_{\mathbf{k}}\left(i \omega_{m}\right)$ by $\Sigma_{\mathbf{k}}\left(i \omega_{m}\right)=\Sigma\left(i \omega_{m}, \theta\right)$ and $\Phi\left(i \omega_{m}, \theta\right)$.

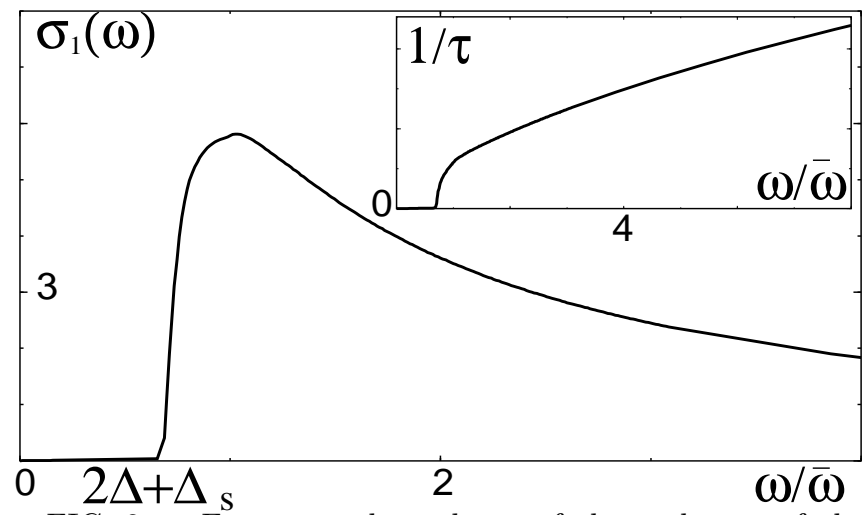

FIG. 2. Frequency dependence of the real part of the optical conductivity $\sigma_{1}(\omega)$ at $T=0$ computed using the self energy and the pairing vertex determined from the Eliashberg equations for $\lambda=1$. The onset of the optical response is $\omega=2 \Delta+\Delta_{s}$. The contributions from nodal regions (not included in calculations) yield a nonzero conductivity at all $\omega$. They also soften the singularity at $\omega=2 \Delta+\Delta_{s}$, but do not eliminate it. Insert - the behavior of the relaxation rate $1 / \tau(\omega)=\left(4 \pi / \omega_{p l}^{2}\right) \operatorname{Re}[1 / \sigma(\omega)]$. The frequency is measured in units $\bar{\omega}$ which sets the overall energy scale in the Eliashberg solution. For $\lambda=1, \Delta=0.204 \bar{\omega}$ and $\Delta_{s}=0.291 \bar{\omega}$.

As our goal is to study the singularities in $\sigma_{1}(\omega)$, we first perform calculations assuming that $\Sigma$ and $\Phi$ are independent on $\theta$ (i.e. that the superconducting gap is flat near the hot spots), and then analyze the results for a true $d$-wave gap. For a flat gap, the momentum integration in Eq. (2) is straightforward. Substituting $k$ integration by integration over $\varepsilon_{k}$, and performing it, we obtain at $T=0$ and $\omega \neq 0$

$$
\Pi_{\sigma}\left(i \omega_{n}\right) \propto \int d \omega_{m}^{\prime} d \theta \frac{\Sigma_{+} \Sigma_{-}+\Phi_{+} \Phi_{-}+D_{+} D_{-}}{D_{+} D_{-}\left(D_{+}+D_{-}\right)}
$$

Here $\Sigma_{ \pm}=\Sigma\left(i \omega_{ \pm}, \theta\right), \Phi_{ \pm}=\Phi\left(i \omega_{ \pm}, \theta\right)$, and $D_{ \pm}=$ $\left(\Phi_{ \pm}^{2}-\Sigma_{ \pm}^{2}\right)^{1 / 2}$, where $\omega_{ \pm}=\omega^{\prime} \pm \omega / 2$. The conductivity is obtained by converting this expression to the real axis [17. The singular piece in $\sigma_{1}(\omega)$ near $2 \Delta+\Delta_{s}$ can be obtained without a precise knowledge of $\Sigma(\omega)$ and $\Phi(\omega)$ : the only information we need is that in a $d$-wave superconductor, $\chi^{\prime \prime}(\mathbf{Q}, \omega)$ has a $\delta$-functional singularity at $\omega=\Delta_{s}$. This is what we found solving a set of three Eliashberg equations. Using this as an input and applying a spectral representation for $\Sigma^{\prime \prime}$ and $\Phi^{\prime \prime}$ (which for a given $\chi^{\prime \prime}(Q, \omega)$ are described by a conventional set of two Eliashberg equations), we obtain that $\Sigma^{\prime \prime}(\omega)$ and $\Phi^{\prime \prime}(\omega)$ are zero up to $\omega=\Delta+\Delta_{s}$, and undergo finite jumps at this frequency. By Kramers-Kronig relation, $\Sigma^{\prime}$ and $\Phi^{\prime}$ diverge as $\left|\log \left(\omega-\omega_{0}\right)\right|$ where $\omega_{0}=\Delta+\Delta_{s}$. The prefactor is the same for $\Sigma^{\prime}$ and $\Phi^{\prime}$. Substituting these forms of $\Sigma(\omega)$ and $\Phi(\omega)$ into (2) we obtain after simple algebra that the conductivity emerges above $2 \Delta+\Delta_{s}$ as $\epsilon^{1 / 2} / \log ^{2} \epsilon$, where $\epsilon=\omega-\left(2 \Delta+\Delta_{s}\right)$. This singularity obviously causes a divergence in the derivatives of the conductivity at $\epsilon=+0$.

In Fig. 2 we show the result for the conductivity obtained by numerically solving Eq.(2) using $\Sigma(\omega)$ and $\Phi(\omega)$ from Ref. [16]. We clearly see the expected threshold at $2 \Delta+\Delta_{s}$. The insert shows the behavior of the relaxation rate $1 / \tau(\omega)=\left(4 \pi / \omega_{p l}^{2}\right) \operatorname{Re}[1 / \sigma(\omega)]$ where $\omega_{p l}$ is the plasma frequency. Observe that $1 / \tau(\omega)$ is linear in $\omega$ over a rather wide frequency range. This agrees with the earlier study of the normal state conductivity [18.

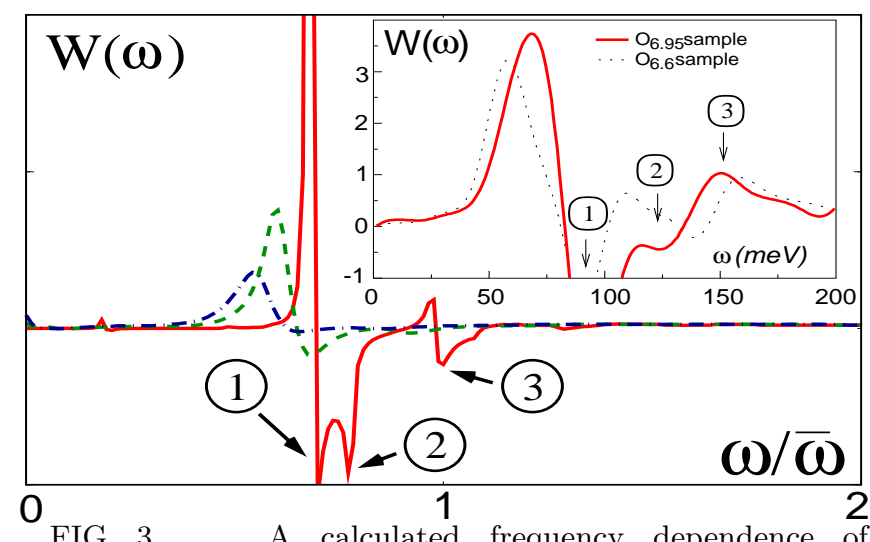

FIG. 3. A calculated frequency dependence of $W(\omega)=\frac{d^{2}}{d^{2} \omega}[\omega \operatorname{Re}[1 / \sigma(\omega)]]$ at $T \rightarrow 0$. This quantity is a sensitive measure of fine structures in the optical response. The locations of the extrema are: $1-2 \Delta+\Delta_{s}, 2-4 \Delta, 3-2 \Delta+2 \Delta_{s}$. Dashed lines are the results are higher $T$. Observe that the maximum shifts to a lower temperature, but the minimum remains at $2 \Delta+\Delta_{s}$. Inset- Experimental results for $W(\omega)$ at low $T$ from Ref. 8]. The position of the deep minimum agrees well with $2 \Delta+\Delta_{s}$. The extrema at higher frequencies are consistent with $4 \Delta$ and $2\left(\Delta+\Delta_{s}\right)$ predicted by the theory.

We next demonstrate that the position of the singularity is not affected by the angular dependence of the gap. Indeed, let the maximum value of the gap correspond to $\theta=0$ and symmetry related points. At deviations from $\theta=0$ both $\Delta$ and $\Delta_{s}$ decrease. The decrease of $\Delta$ is obvious, the decrease of $\Delta_{s}$ is due to the fact that resonance is a feedback from superconductivity, and its frequency scales as $(\Delta(\theta))^{1 / 2}$. Since both $\Delta$ and $\Delta_{s}$ are maximal at a hot spot, we can expand $\omega_{0}(\theta)=\Delta(\theta)+\Delta_{s}(\theta)$ as $\omega_{0}(\theta)=\omega_{0}-a \theta^{2}$, where $a>0$. The singular pieces in $\Sigma(\omega)$ and $\Phi(\omega)$ then behave as $\left|\log \left(\omega_{0}-\omega-a \theta^{2}\right)\right|$. Substituting these forms into (2) and integrating over $\theta$, we find that the conductivity itself and its first derivative are continuous at $\omega=2 \Delta+\Delta_{s}$, but the second derivative of the conductivity diverges as $d^{2} \sigma / d \omega^{2} \propto 1 /\left(|\epsilon| \log ^{2} \epsilon\right)$ where, we remind, $\epsilon=\omega-\left(2 \Delta+\Delta_{s}\right)$. We see that the singularity is weakened by the angular dependence of the 
gap, but it is still located at exactly $2 \Delta+\Delta_{s}$.

The same reasoning is also applied to a region near $4 \Delta$. We found that the singularity at $4 \Delta$ is also weakened by the angular dependence of the gap, but is not shifted and still should show up in the second derivative of the conductivity.

We now discuss the second derivative of the conductivity in more detail. In Fig. 3 we present our numerical results for $W(\omega)=\frac{d^{2}}{d^{2} \omega}\left(\omega \operatorname{Re} \sigma^{-1}(\omega)\right)$ which effectively measures second derivative of conductivity (we followed Ref [8] and used the same $W(\omega)$ as for phonon superconductors). We clearly see that there is a sharp maximum in $W(\omega)$ near $2 \Delta+\Delta_{s}$ followed by a deep minimum. We also see that $W(\omega)$ has extra extrema at $4 \Delta$ and, also, at $2 \omega_{0}=2 \Delta+2 \Delta_{s}$. The last peak is a secondary effect due to a singularity in $\Sigma(\omega)$ at $\omega=\omega_{0}: \sigma_{1}(\omega)$ is singular when the frequencies of both fermions in the polarization bubble exceed $\omega_{0}$.

The experimental result for $W(\omega)$ in YBCO is shown in the insert. We see that the theoretical and experimental plots of $W(\omega)$ look rather similar, and the relative intensities of the peaks are at least qualitatively consistent with the theory. By the reasons which we display below, we identify $2 \Delta+\Delta_{s}$ with the deep minimum in $W(\omega)$. This yields $2 \Delta+\Delta_{s} \approx 100 \mathrm{meV}$. Identifying the extra extrema in the experimental $W(\omega)$ with $4 \Delta$ and $2 \Delta+2 \Delta_{s}$, respectively, we obtain $4 \Delta \sim 130 \mathrm{meV}$, and $2 \Delta+2 \Delta_{s} \sim 150 \mathrm{meV}$. We see that three sets of data are self-consistent and yield $\Delta \sim 30 \mathrm{meV}$ and $\Delta_{s} \sim 40-45 \mathrm{meV}$. The value of $\Delta$ is in good agreement with tunneling measurements [20], and $\Delta_{s}$ agrees well with the resonance frequency extracted from neutron measurements [21]. We caution, however, that determination of a second derivative of a measured quantity is a very subtle procedure. The good agreement between our theory and the experiment is promising but have to be verified in further experimental studies. Nevertheless, our calculation clearly demonstrates the presence and observability of these "higher harmonics" of the optical response at $4 \Delta$ and $2 \Delta+2 \Delta_{s}$.

So far we considered only the singular part of $\sigma_{1}(\omega)$. In Fig. 1 we compare our results for $\sigma_{1}(\omega)$ (ignoring the contributions from the nodes) directly with the experimental data by Puchkov et al. 19] for optimally doped $\mathrm{YBa}_{2} \mathrm{Cu}_{3} \mathrm{O}_{6+\delta}$. We used $\lambda=1$ and the overall energy scale $\bar{\omega}$ which yield $\Delta \sim 30 \mathrm{meV}$ and $\Delta_{s} \sim 45 \mathrm{meV}$ as the solution of the Eliashberg set, and also $\omega_{p}=$ $1.2 \times 10^{4} \mathrm{~cm}^{-1}$, similar to that in 19$]$. We see that the frequency dependence of the conductivity at high frequencies agrees well with the data. The measured conductivity drops at about $100 \mathrm{meV}$ in rough agreement with $2 \Delta+\Delta_{s} \approx 100 \mathrm{meV}$ in our theory. As in earlier studies [14, 18], to match the magnitude of the conductivity, we had to add the constant $7 \times 10^{-4} \Omega \mathrm{cm}$ to $\left(\sigma_{1}(\omega)\right)^{-1}$. We view the good agreement between theory and experi- ment at $\omega>2 \Delta+\Delta_{s}$ is predominantly an indication that the momentum dependence of the fermionic dynamics becomes irrelevant at high frequencies, and fermions from all over the Fermi surface behave as if they were at hot spots. The insert to Fig. 1 shows $\sigma_{1}^{-1}(\omega)$. We see that it is linear over a substantial frequency range.

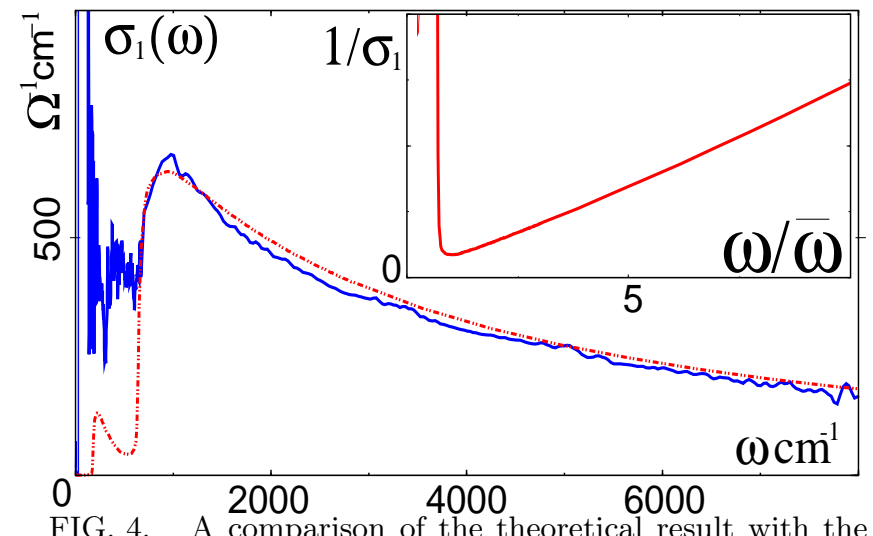

FIG. 4. A comparison of the theoretical result with the experimental data of Puchkov et al. [19]. The substructure in the theoretical $\sigma_{1}$ at low frequencies is an artifact. Insert the behavior of $\sigma_{1}^{-1}(\omega)$.

We emphasize, however, that this linearity is only an intermediate asymptotic. At the highest $\omega$, our theory yields $\sigma_{1}(\omega) \propto \omega^{-1 / 2}$. The lower boundary for $\omega^{-1 / 2}$ behavior decreases with increasing $\lambda$. For optimally doped $B i 2212, \lambda$ is somewhat larger than in $Y B C O$ as $\Delta \approx \Delta_{s}$, and we expect a more pronounced $\omega^{-1 / 2}$ behavior at high frequencies. This trend is consistent with the data of Ref. [22]. This issue, however, requires further study as $\sigma_{1}(\omega) \propto \omega^{-1 / 2}$ at intermediate frequencies was also obtained in Ref. [14 assuming a strongly momentum dependent scattering rate.

Finally, we comment on the position of the $2 \Delta+\Delta_{s}$ peak and compare our results with those by Carbotte et al. [8]. Theoretically, at $T=0$ and in clean limit, the maximum and minimum in $W(\omega)$ are at the same frequency. We found, however, that at finite $T$, they quickly move apart. We present the theoretical temperature dependence of $W(\omega)$ in Fig 3. Carbotte et al. [8] focused on the maximum in $W(\omega)$ and argued that it is located at $\Delta+\Delta_{s}$ instead of $2 \Delta+\Delta_{s}$. We also found that the maximum in $W(\omega)$ shifts to a lower frequency with increasing temperature, already at $T$ where the temperature dependence of the gap may be neglected. On the other hand, the minimum in $W(\omega)$ moves very little with increasing $T$ and virtually remains at the same frequency as at $T=0$. This is our reasoning to use the minimum in $W(\omega)$ as a much more reliable feature for the comparison with experiments. This reasoning is in agreement with recent conductivity data on optimally doped Bi2212 22] - $W(\omega)$ extracted from these data shows strong downturn variation of the maximum in $W(\omega)$ with increasing temperature, but the minimum in $W(\omega)$ is located at 
around $110 \mathrm{meV}$ for all temperatures.

Finally, we briefly consider whether one can extract a resonance spin frequency from the measurements of the Raman intensity in a $d$-wave superconductor. The Raman intensity is given by [23]

$$
R(\omega)=\operatorname{Im} \int d \theta V^{2}(\theta) \Pi_{R}(\omega, \theta)
$$

where $V(\theta)$ is Raman matrix element, and $\Pi_{R}$ is same bubble as for conductivity, but with a different sign of the anomalous $F F$ term. The latter is a consequence of the fact that Raman vertices are scalar and do not change sign under $k->-k$. Performing the integration over quasiparticle energies in the same way as for conductivity we obtain in Matsubara frequencies [25]

$$
\Pi_{\sigma}\left(i \omega_{n}\right) \propto \int d \omega_{m}^{\prime} d \theta \frac{\Sigma_{+} \Sigma_{-}-\Phi_{+} \Phi_{-}+D_{+} D_{-}}{D_{+} D_{-}\left(D_{+}+D_{-}\right)}
$$

For mostly studied $B_{1 g}$ scattering, the Raman vertex has the same angular dependence as the $d$-wave gap, i.e., $V(\theta) \propto \cos (2 \theta)$ [23]24]. Straightforward computations then show that for a $d$-wave gas, $R(\omega) \propto \omega^{3}$ at low frequencies [24], and diverges as $\omega$ approaches $2 \Delta$ first as $|\omega-2 \Delta|^{1 / 2}$, and then, in the immediate vicinity of $2 \Delta$, as $\log |\omega-2 \Delta|$ [24,26]. At larger frequencies $R(\omega)$ gradually decreases. At strong coupling, we performed the same analysis as for conductivity and found that the sign change of the $F F$ term in the bubble, compared to that for conductivity, has a drastic effect: near $2 \Delta+\Delta_{s}$, singular contributions from $\Sigma_{+} \Sigma_{-}$and $\Phi_{+} \Phi_{-}$terms in Eq.(17) cancel each other. As a result, for a flat gap, only the second derivative of $R(\omega)$ diverges at $2 \Delta+\Delta_{s}$. For a quadratic variation of a gap near its maximum, the singularity is even weaker and shows up only in the third derivative of $R(\omega)$. Obviously, this is a much weaker effect than that for conductivity, and its determination requires a high quality of the experiment. Notice, however, that due to the closeness of hot spots to $(0, \pi)$ and related points, at which $v_{F}$ vanishes, the actual smearing of the singularity due to the angular integration may be less drastic than in our theory and the singularity in $R(\omega)$ at $2 \Delta+\Delta_{s}$ may possibly be extracted from the data.

To conclude, in this paper we examined the singularities in the optical conductivity in a $d$-wave superconductors assuming that the pairing is mediated by overdamped spin fluctuations. We argued that $\sigma_{1}(\omega)$ should have singularities at $2 \Delta+\Delta_{s}, 4 \Delta$ and $2 \Delta+2 \Delta_{s}$, where $\Delta$ is the maximum value of the $d$-wave gap, and $\Delta_{s}<2 \Delta$ is the resonance spin frequency. The experimental detection of these singularities would be a strong argument in favor of the magnetic scenario. We argued that there is a good possibility that all three singularities have actually been detected in recent data on $Y B C O$.

It is our pleasure to thank D. N. Basov, G. Blumberg, J.C. Campuzano, J. Carbotte, P. Coleman, O. Dolgov,
P. Johnson, M. Norman, D. Pines, E. Schachinger, S. Shulga and J. Zasadzinski for useful conversations. We are also thankful to D. N. Basov, C. Homes, M. Strongin and J. Tu for sharing unpublished results with us. The research was supported by NSF DMR-9979749 (Ar. A and A. Ch.) and by the Ames Laboratory, operated for the U.S. DoE by Iowa State University under contract No. W-7405-Eng-82 (J.S).

[1] D. A. Wollmann, D. J. Van Harlingen, W. C. Lee, D. M. Ginsberg, and A. J. Leggett, Phys. Rev. Lett. 71, 2134 (1993); C. C. Tsuei, J. R. Kirtley, C. C. Chi, Lock See Yu-Jahnes, A. Gupta, T. Shaw, J. Z. Sun, and M. B. Ketchen ibid 73, 593 (1994).

[2] Farnworth and Timusk, Phys. Rev. B bf 10, 2799 (1974); J. P. Carbotte, Phys. Lett. A 245 , 172 (1998).

[3] D.J. Scalapino, Phys. Rep. 250, 329 (1995); D. Pines, Z. Phys. B 103, 129 (1997) and references therein.

[4] M. R. Norman et al, Phys. Rev. Lett 79, 3506 (1997); Z-X. Shen and J.R. Schrieffer, Phys. Rev. Lett. 78, 1771 (1997); M. R. Norman and H. Ding, Phys. Rev. B 57, R11089 (1998).

[5] A. Chubukov, Europhys. Lett. 44, 655 (1997); Ar. Abanov and A. Chubukov, Phys. Rev. Lett., 83, 1652 (1999), cond-mat/0002122.

[6] T. Holstein, Phys. Rev. 96, 535 (1954); P.B. Allen, Phys. Rev. B 3, 305 (1971). For recent detailed review see S.V. Shulga, in Proceedings of the Albena Worksop, 1998, Kluwer Publishers, Dortrecht, p.323 (2001).

[7] J. F. Zasadzinski, L. Ozyuzer, N. Miyakawa, K.E. Gray, D.G. Hinks and C Kendzora, submitted to PRL

[8] J. P. Carbotte, E. Schachinger, D. N. Basov, Nature (London) 401, 354 (1999).

[9] A. Chainani et al., Phys. Rev. Lett. 85, 1966 (2000).

[10] P. Littlewood and C.M. Varma, Phys. Rev. B 46, 405 (1992).

[11] G.D. Mahan, Many-Particle Physics, Plenum Press, 1990.

[12] S. M. Quinlan, P. J. Hirschfeld, D. J. Scalapino, Phys. Rev. B 53, 8575 (1996).

[13] B. P. Stojkovic and D. Pines, Phys. Rev. B 56, 11931 (1997).

[14] L. B. Ioffe and A. J. Millis, Phys. Rev B 58, 11631 (1998), see also A. T. Zheleznyak, V. Yakovenko, H. D. Drew, and I. I. Mazin, Phys. Rev. B 57, 3089 (1998).

[15] Ar. Abanov, A. Chubukov, and A. M. Finkel'stein, condmat/9911445

[16] Ar. Abanov, A. Chubukov, and J. Schmalian, condmat/0005163.

[17] R. Combescot et al, Phys. Rev. B 53, 2739 (1996).

[18] R. Haslinger, Ar. Abanov, and A. Chubukov Phys. Rev. B 63, 020503(R) (2001).

[19] A. V. Puchkov et al J. Phys. Chem. Solids 59, 1907 (1998); D. N. Basov R. Liang, B. Dabrovski, D. A. Bonn, W. N. Hardy and T. Timusk, Phys. Rev. Lett. 77, 4090 
(1996); D.N, Basov et al, unpublished.

[20] N. Miyakawa et al, Phys. Rev. Lett. 83, 1018 (1999).

[21] H.F. Fong et al, Phys. Rev. B 54, 6708 (1996); H.F. Fong et al, Nature 398, 588 (1999); P. Dai et al, Science 284, 1344 (1999).

[22] C. Homes, M. Strongin, and J. Tu, private communication.

[23] M.V. Klein and S.B. Dierker, Phys. Rev. B 29, 4976 (1984).

[24] T.P. Devereaux et al., Phys. Rev. Lett. 72, 396 (1994); Phys. Rev. B 54, 12523 (1996).

[25] see e.g. A. Chubukov, G. Blumberg, and D. Morr, Solid State Comm. 112, 183 (1999).

[26] N. Gemelke, A. Abanov and A. Chubukov, Phys. Rev. B 61, R6467 (2000). 